\title{
Botanische Notizen.
}

(Aus einem Schreihen des IIru. Apotb. II a mpe zu Blankenburg an Dr. Bley.)

Um die Mitte Scptembers bin ich noch auf dem Brocken gewesen und von meiner Excursion befriedigt: denn ich habe der Flora germanica einen neuen Bürger zuführen können, nämlich ein Moos. Orthotrichum Drammondii Hook, welches bisher nur in der subarctischen Zone Norwegen, Schottland und Canada gefunden ist. Im Ganzen schien mir die Vegetation der obern Gebirge noch an den Nachwehen von 1842 zu leiden. - Vor einigen Tagen erhielt ich cine kleire Parthic fingerdicke Wurzeln von Rheum australe Don, welche in Deutschland versuchsweise cultivirt sind; eine vergleichende Untersuchung wäre vielleicht nicht ohne Interesse *).

\section{Einige Bemerkungen uiber Polygala amara Jacq. und die dazu gehörigen Abarten;}

volt

Moritz Osswald,

Ilofapothelser in Eisenach.

Durch Hrn. Prof. Koch in Erlangen veranlasst, untersuchte ich folgende Polygala-Arten, die-sämmtlich bei Arnstadt vorkommen, hinsichtlich ihres bittern Geschmacks und genauen botanischen Unterschiedes.

1) Polygala amara Jacq. amarella Rchb. kommt häufig nur auf Kalkboden in lichten Laubwäldern vor, und unterscheidet sich von der folgenden Art durch dichte rosettenförmige Wurzelblätter und grössere dunkelblane Blumen. Das Decoct, aus der ganzen Pllanze bereitet, die zur Blüthenzeit gesammelt war, hat einen anhaltenden, bittern Geschmack.

*) Im Fall IIr. Collegc II a m p dic gewiss nicht uninteressante Untersuchung nicht selbst volnehmen will, bin ich gern zu selbiger bereit.

Bley.

Arch. d. Pharm. LXXXVII. Bds. 1. IIft. 
2) Polygala austriaca $\mathrm{Cr}$., zu dieser rechnet $\mathrm{Koch}$ in seiner Synopsis Polygala uliginosa Rchb. und das wohl mit Recht; denn untersucht man mehre Exemplare von ein und demselben Standorte (wie z. B. die auf feuchten Wiesen wachsenden bei Arnstadı unter der Eremitage), so findet man, dass die Stengelblätter bald schmäler, bald mehr verkehrt eiförmig, dass die Kapseln bald rundlicher, bald mehr verkehrt eiförmig, und dass endlich die Flügel bald so breit als die Kapseln, bald um die Hälfte schmäler, und dass sie bald nur so lang sind als die Kapseln, bald merklich länger.

Die Polygala uliginosa Rchb. ist eigentlich wohl nichts anderes, als die auf feuchten Wiesen wachsende $P$. austriaca $C r$. Sie kommt gewöhnlich mit gesättigter blauer Farbe vor, ist aber nicht so bitter, als $P$. amara Jacq., ja ich habe bemerkt, dass dieselbe, je feuchter sie steht, nämlich die an Wassergräben, mehr einen krautartigen, als bittern Geschmack besitzt; diese düfte nun nicht zum medicinischen Gebrauch gecignet scin. Auffallend ist es, dass die nach Prof. Ko ch bei Erlangen auf Wiesen vorkommende $\mathrm{P}$. austriaca $\mathrm{Cr}$. weissliche Blithen hat, selten gesättigt blaue, und dass sie dort als sehr bitter und wirksam von den Apothekern gesammelt wird.

Diese Art wächst nicht allein auf Wiesen und ist nicht. wie Koch und D. Fl. sagt. bluss den Niederungen eigen, sondern kommt auf mehr oder weniger beschattetem Kalkboden vor, und dann ist sie gewöhnlich mit weisslicher und bläulichweisser Farbe vorherrschend zu finden. Nach Angabe des Hrn. Pastor Schönheit in Singen ist sio über einen grossen Theil von Thüringen verbreitet. Lnd diese auf Bergen vorkommende Pflanze liefert ein ebenso bitteres Decoct, als P. amara Jacq.

3) Polygala amblyptera Rohb. ist nach meinem Dafürhalten nichts anderes, als eine Form von P. amara Jacq. ; sie wächst in Gemeinschaft derselben auf Kalkboden in Laubwäldern, und die Exemplare, die ich der Güte der Herren Apotheker Lucas und Dr. Nicolai in Arnstadt verdanke - selbst konnte ich sie nie auffinden - sind nur durch etwas breitere Fliggel, welche fast so breit als 
die Kapseln sind, verschieden. Unter demselben Namen besitze ich Exemplare vom Rheine an der Ems, (?) welche diesen Unterschied nicht haben. - Uebrigens liefert diese Art ein eben so bitteres Decoct, als P. amara Jacq.

Will man zu $P$. amara Jacq. die $P$. austriaca $C r$. und uliginosa Rchb. rechnen, so ist $P$. amara Jacq. der älteste Name, denn die Enumerat. stirp. Vindob. erschien 1762. Der Name austriaca Cr. stammt aus dem Werke Crantz Stirp. austriac. vom Jahre 1769.

\section{Nachschrift von E. G. Hornung.}

Der geneigten Aufforderung des Hrn. Collegen Osswald gern genügend, meine Bemerkungen zu obigem Aufsatze zu machen, erlaube ich mir, demselben einige Zeilen anzureihen.

$\mathrm{Zu}$ meinem Bedauern habe ich bisher Polygala amara so wenig als eine ihrer Varietäten in unserer nähern Umgebung aufgefunden, und konnte daher auch in neueren Zeiten keine Beobachtungen über den bald vorhandenen, bald mangelnden bittern Geschmack derselben machen.

Ich gestehe auch offen, dass ich hierauf keinen so grossen Werth legen möchte. Ich habe meine Polygala amara und deren Varietäten aus verschiedenen Standorten geerntet, aber keine ganz geschmacklos gefunden, wohl aber bemerkt, dass die Pflanze in Betreff der Bitterkeit nicht weniger variirt, als in den übrigen Merkmalen, durch dic man ihre verschiedenen Formen -zu unterscheiden sich bemuiht hat. Sowie man aber in allen diesen Formen sogleich die P. amara wieder erkennt, so scheint mir auch der eigenthümliche bittere Geschmack, wenigstens bei der getrockneten Pflanze, immer derselbe, wenn auch zuweilen etwas schwach. Dasselbe spricht auch Koch in seinem Taschenbuch der Deutschen und Schweizer Flora S. 69 aus : Kommt sehr bitter und fast geschmacklos vor." Ja, ich vermuthe, dass die auf feuchten Wiesen vorkommende in frischem Zustande geschmacklose Pflanze durch das Trocknen ebenfalls bitter werde, denn ich besitze einige Excmplare von meinem Freunde Lucas in Arnstadt, bei 
denen er noch bemerkt hat: „diese Polygala kommt hier auf feuchten Wiesen an Bachründern vor, ich muss sie ihrer Geschmacklosigkeit wegen für $P$. uliginosa halten. Ausser ihrer Geschmacklosigkeit finde ich übrigens keinen Entersehied von der hier im Walde vorkommenden $P$. amara oder $P$. amblyptera?" Ich finde aber diese beigefïgten Exemplare der Wiesenpllanze keineswegs geschmacklos, sondern fast noch bitterer als die der Waldpflanze. So sind auch meine Exemplare der $P$. austriaca von Wien, Linz und aus der Schweiz bitter, aber die der $P$. uliginosa von Leipzig und Frankenhausen keineswegs geschmacklos, ja zum Theil, wie auch solche von Breslau, sehr bitter. Auffallend wenig bitter ist dagegen die ächte P. amara (nach Reichenbach) von Frankenhausen, während eine $P$. amara, die ich bei Coburg, und wenn ich nicht irre, auf feuchten Wiesen sammelte, sehr bitter ist; die sogenannte P. amblyptera i:t es wenig, alser noch weniger dic $\boldsymbol{P}$. alpestris von der Pasterze und vom Jura. Ich stimme übrigens Hin. Hofrall ko ch vollkommen bei, alle diese Pflanzen nur als Formen der P. amara zu betrachten, denn dic aufgesuchten Unterscheidungsmerkmale sind zu wenig bestindlig; Standort, Alter und Entwickelung der Pflanze sind dabei von grossem Einflusse. Meine oben aufgestellte Vermuthung, dass die im frischen Zustande gesclimacklose Pflanze durch das Trocknen bitter werde, verdient immerhin eine Prüfung, welche mcine werthen Collegen, in deren Nähe P. amara vorkömmt, wohl ibernchmen und die Erfolge in dem Archive mittheilen werden.

Die im vorstehenden Aufsatze erwähnte Arnstädter Pflanze sollte man jedoch nicht ferner P. amblyptera Rchb. nennen, denn dagegen hat R eich en bach selbst protestirt, indem er in der Flora excursoria p. 35t sagt: "Semper falsa Specimina misit Hornung" und es scheint mir auch selbst, dass bei dieser Pflanze, die merkwürdigerweise verdammt zu scin scheint, fortwährend Verirrungen hervorzurufen, irgendwo ein Irrthum untergelaufen sein mag. Ich erhielt dieselbe von meinem Freunde $L$ a cas in grosser Anzahl mit der Versicherung, dass das dieselbe 
Pflanze sei, die er an Hrn. Hofrath Reichenbach gesendet habe, und obschon sie nicht recht zu Reich en b a ch's Abbildung Icon. I. No. 50. passen wollte, so suchte ich meine $\mathrm{Z}$ weifel durch die Vermuthung zu beschwichtigen, dass der von Reichenbach abgebildeto Ast einer üppigen, melir entwickelten Pflanze angehört habe, während meine Exemplare noch ziemlich jugendlich waren, und das um so mehr, da mir mein Freund Lucas wiederholt versicherte, keine andere Polygala aus dieser Gruppe um Arnstadt auffinden zu können, und Reichenbach selbst scine amblyptera in der Iconographie nur mit der "schr ähnlichen " amarella $C r$. (der amara $L$.) vergleicht. Da nun aber meine Lucas'sche Pflanze .von Reichenbach nicht als die seinige anerkannt worden ist, so kann sie auch nicht ferner unter dessen Autorität ausgegeben werden, sondern mag, wenn man einmal die Varietäten der P. amara bezeichnen will, als P. amara am:blyptera Koch aufgefuhrt werden; als eigne Arten aber wird im Ernste wohl Niemand melr die Formen der $\boldsymbol{P}$. amara aufstellen.

Nach diesen Erörterungen hätten wir nun die räthselhafte $\boldsymbol{P}$. amblyptera Rchb. wohl wo anders zu suchen. Räthselhaft darf ich sie wohl nennen, da selbst der tüchtige Dresdener Botaniker, Heynhold, sie noch nicht zu kennen scheint, indem er sie in sciner Flora von Sachsen 1842 noch $n$ ach Koch's Synopsis als $P$ : amara $\beta$ amblyptera auffuihrt. Wir wollen deshalb an die Quolle zurückgehen, nämlich zu Roichenbach's Iconographie. Ilier finden wir auf Tafel 24. unter No. 50. dessen var. $\alpha$ glabra und unter No. 51. dessen var $\beta$ pubescens, und S. 26 des Textes citirt derselbe zu $\alpha$ glabra, seiner Arnstädter Pflanze, P. buxi minoris folio Vaill. t. XXXII. f. 2. und zu $\beta$ pubescens die P. pubescens Rhode, nicaeensis Risso, vulgaris $\beta$ pubescens $D C$. Dass P. nicaeensis, oder die var. $\beta$ aber nicht hierher gehört oder eigentlich nicht die ächte P. amblyptera Rchb. Icon. und flora excurs. sein kann, hat Ko ch in seiner Flora V. S. 69 schon ausgespirnchen, und ich stimme demselben vollkommen bei. Der langgestielte 
Fruchtknoten würde allein schon zum genügenden Unterschiede hinreichen, diesen aber erwähnt Reich enbach eben so wenig in der Iconographie, als in der Flora excursoria; auch gehört der auf Taf. 24. unter $B$ abgebildete Fruchtknoten nicht der Nizzaer Pflanze, sondern der Thüringer an. Diese var. $\beta$, welche Reich enbach selbst in der flora exc. nur noch mit einem Fragezeichen anfiuhrt, muss daher ganz ausgeschlossen werden. Dass dem sonst so scharfsichtigen Reichenbach dieser lange Stiel des Fruchtknotens entgehen konnte, ist in der That auffallend und ich würde anstehen, mich mit so viel Sicherheit über diese Pflanze auszusprechen, besässe ich nicht unter anderm ein von Reich enbach selbst bestimmtes Exemplar von Nizza, welches ich der Güte meines verehrten Freundes $\mathrm{K}$ o ch verdanke. Die Pflanze variirt, beiläufig gesagt, in Haltung nicht unbedeutend und kömmt auch im Süden glatt und behaart vor.

Es bliebe uns demnach nur noch die var. $\alpha$ (No. 50. f. B.) der Iconographie und das Citat aus Vaillant übrig. Die Abbildung No. 50 deutet aber offenbar auf einige Verwandtschaft mit $\boldsymbol{P}$. vulgaris und dafür spricht auch die Stellung, die ihr Reich enbach giebt. In der Iconographie nämlich bringt er sie zwischen $\boldsymbol{P}$. oxyptera und vulgaris, und in der Flora excursoria hinter P. oxyptera. Das Citat aus Vaillant aber, welches ich freilich nicht vergleichen kann, scheint zweifelhaft, wenn es nicht vielleicht gerade geeignet ist, uns zum richtigen Erkenntniss der Pflanze zu führen. Dieselbe t. 32. f. 2 von Vaill. bot. paris., welche Reichenbach bei seiner Pflanze citirt, zieht Ko ch nämlich in seiner Flora V. S.74 zu P. calcarea Schulz., auch hat $\mathrm{S}$ chulz seine $\boldsymbol{P}$. calcarea anfangs selbst für $\boldsymbol{P}$. amblyptera Rchb. gehalten und ist vielleicht nur durch meine Arnstädter Exemplare irre geleitet worden. Ob dieses der Fall ist oder ob ihn andere Gründe bestimmten, seine anfängliche Ansicht aufzugeben, dariber vermag ich noch keinen Aufschluss zu geben, aber es ist mir nicht unwahrscheinlich, dass wir in der $\boldsymbol{P}$. calcarea die ächte $\boldsymbol{P}$. amblyplera zu suchen haben. Die Flora Saxonica von Rei- 
chenbach ist mir leider nicht znr Hand, um nachschlagen zu können, ob er daselbst vielleicht die calcarea zu seiner amblyptera zieht. Wuinschenswerth ist es jedoch, diese Pflanze endlich aufgeklärt zu sehen. Indem ich nun durch die vertheilten Exemplare vielleicht dazu beigetragen habe, die Verwirrung in Betreff dieser Pflanze zu vermehren, ergreife ich jetzt auch mit Vergnuigen die Feder, um eine Auflösung dieser Wirren wo möglich herbeizuführen. Ich ersuche deshalb meinen Freund L u cas, seine Forschungen an den Kalkbergen bei Arnstadt zu erneuern; denn wenn jene Pflanze wirklich bei Arnstadt gesammelt war, so ist es wenigstens wahrscheinlich, dass sie auch wieder aufgefunden wird. Nach Heynhold's Flora von Sachsen soll $P$. calcarea auch bei Jena vorkommen. Kürzer würden wir aber ans Ziel gelangen, wenn Hr. Hofrath Reichenbach uns belehren, oder Hr. Dr. Schulz nach diesen Erörterungen den Gegenstand nochmals aufnehmen wollte. Bestätigt sich meine Vermuthung, so wäre es vielleicht besser, den Namen amblyptera ganz aufzugeben.

Anhang.

Schon war obige Erörterung für die Absendung eịngepackt, und ich suchte nun nochmals vergeblich in der Flora nach einem Aufsatze des Hrn. Hofraths Koch*), in welchem derselbe, wenn ich nicht irre, über $P$. calcarea, sowie über die berührte Bitterkeit der P. amara spricht, als mir in den Jahrgängen 1837. und 1838. die beiden Aufsätze des Hrn. Dr. Schulz in die Hände kamen, welche, namentlich der letztere, mir den gewünschten Aufschluss gaben. Er führt S. 642 nämlich unter den Synonymen seiner $P$. calcarea auch Folgendes auf: "P. amblyptera Schul (etc.) Hornung!, nec Reichenbach!, quae ad P. nicaeensem Risso, Speciem omnino diversam, pertinet. Ferner sagt er noch: "dass Reich enbach's amblyptera nicht unsere Pflanze sei, geht daraus deutlich hervor, dass li eichenbach in der Flora germ. excurs. 2101 sagt: Semper falsa etc.

*) Vielleicht befindet er sich in Jnhrgange 1842, der mir eben nicht zur Jland ist. 
Das bestätigt nun meine Vermuthung, dass Schulz nur durch meine Arnstaidter Pflanze irregefuhrt, sciner amblyptera einen neuen Namen gab, es bestärkt mich aber auch in meiner oben ausgesprochenen Ansicht, dass wir gerade in dieser Pflanze die ächte amblyptera $R$. zu suchen haben, wenn auch scheinbar ein neues Element der Verwirrung in den Kreis tritt, nämlich dass Schulz meine Arnstädter Pllanze zu seiner $\boldsymbol{P}$. calcarea zicht. Offenbar ist er aber hier im Irrthum, denn wenn ich auch kein Schulz'sches Originalexemplar zur Vergleichung vor mir habe, so lässt doch die nach solchen entworfene ausfuhrliche Beschreilung Koch's kcinen Zweifel darüber, dass meine Pflanze von $\boldsymbol{P}$. calcarea wesentlich verschieden sei; sie gehört in jedem Bezuge der $\boldsymbol{P}$. amara an. Bedürfte es noch eines besondern Beweises, so möchte wohl der genïgen, dass Koch, nachdem er auf S. 74 seiner Flora die $\boldsymbol{P}$. calcarea sorgfälig erörtert hat und nur Zweibrücken als Fundort aufführt, auf S. 76 meine Arnstädtor Pflanze, wolche er in Mehrzahl von mir besitzt, zu $\boldsymbol{P}$. amara bringt und sie auch mit Recht nicht cinmal mehr als Varictait sondert.

Ich bin gern erbötig, Hrn. Dr. Schulz selbst Exemplare meines alten Vorraths dieser Streitpflanze mitzutheilen, und halte mich im Voraus überzeugt, dass er nach erneuerter Prüfung meiner Meinung beitrelen wird und wir uns friedlich die Hände reichen werden. Auch $\mathrm{Hr}$. Hofrath $R$ eichenbach dürfte versöhnt werden, wenn es mir gelingt, seine vielgeprüfte (valde vexata? $\boldsymbol{P}$. amblyptera wieder in ihre Rechte einzusetzen. 